\title{
Zero-velocity solitons in high-index photonic crystal fibers
}

\author{
Lægsgaard, Jesper
}

\section{Published in:}

Optical Society of America. Journal B: Optical Physics

Link to article, DOI:

10.1364/JOSAB.28.000037

Publication date:

2011

\section{Document Version}

Publisher's PDF, also known as Version of record

Link back to DTU Orbit

\section{Citation (APA):}

Lægsgaard, J. (2011). Zero-velocity solitons in high-index photonic crystal fibers. Optical Society of America. Journal B: Optical Physics, 28(1), 37-44. https://doi.org/10.1364/JOSAB.28.000037

\section{General rights}

Copyright and moral rights for the publications made accessible in the public portal are retained by the authors and/or other copyright owners and it is a condition of accessing publications that users recognise and abide by the legal requirements associated with these rights.

- Users may download and print one copy of any publication from the public portal for the purpose of private study or research.

- You may not further distribute the material or use it for any profit-making activity or commercial gain

- You may freely distribute the URL identifying the publication in the public portal

If you believe that this document breaches copyright please contact us providing details, and we will remove access to the work immediately and investigate your claim. 


\title{
Zero-velocity solitons in high-index photonic crystal fibers
}

\author{
Jesper Lægsgaard \\ DTU Fotonik, Technical University of Denmark, Building 343, DK-2800 Kgs. Lyngby, Denmark (jlag@fotonik.dtu.dk)
}

Received August 24, 2010; revised November 2, 2010; accepted November 3, 2010;

posted November 4, 2010 (Doc. ID 133880); published December 10, 2010

Nonlinear propagation in slow-light states of high-index photonic crystal fibers (PCFs) is studied numerically. To avoid divergencies in dispersion and nonlinear parameters around the zero-velocity mode, a time-propagating generalized nonlinear Schrödinger equation is formulated. Calculated slow-light modes in a solid core chalcogenide PCF are used to parameterize the model, which is shown to support standing and moving spatial solitons. Inclusion of Raman scattering slows down moving solitons exponentially, so that the zero-velocity soliton becomes an attractor state. An analytical expression for the deceleration rate that compares favorably with the numerical results is derived. Collisions of successive solitons due to the Raman deceleration are studied numerically, and it is found that the soliton interaction is mostly repulsive, as expected from the established theory of fiber solitons. (c) 2010 Optical Society of America

OCIS codes: $\quad 060.4005,060.4370,060.5295,060.5530,190.5650,190.6135$.

\section{INTRODUCTION}

Slow-light states in photonic bandgap (PBG) structures have been extensively studied in the last few years, due to the prospects of all-optical signal buffering or enhancement of lightmatter interactions (for a recent review, see [1] ). While the fabricated slow-light waveguides have so far been realized in planar photonic crystals, a theoretical possibility exists of achieving slow-light guidance in high-index optical fibers as well [2]. By fabricating microstructured fibers of high-index materials, a complete PBG in the plane of the fiber cross section can be opened up, which will allow guidance of modes with propagation constants around zero. By very general arguments of time-reversal symmetry, such modes will have a vanishing group velocity when the propagation constant, $\beta$, becomes zero. It was recently pointed out that the refractive index of $\mathrm{As}_{2} \mathrm{Se}_{3}$ chalcogenide glass is high enough to open up robust in-plane bandgaps in realistic fiber geometries [3]. Since fiber drawing from such glasses is well established [ㄴ] $]$, the fabrication of slow-mode fibers seems a realistic opportunity, and it is therefore interesting to study their properties to identify possible applications. Applications for gas sensing with enhanced sensitivity was studied theoretically in a recent paper [5]. The purpose of the present work is to set up a general framework for modeling the nonlinear properties of slow-mode fibers and use it to investigate the propagation of fundamental solitons in the slow state.

Traditionally, nonlinear pulse propagation in optical fibers has been modeled by $z$-stepping schemes, in which the pulse profile as a function of time is specified at the input end and then propagated along the fiber by a first-order differential equation known as the nonlinear Schrödinger equation (NLSE) and its generalizations. In recent years, substantial efforts have been put into developing such schemes to deal with the challenges of modern fiber technology, including full-

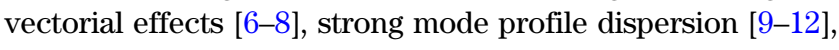
and ultrashort pulses [10]. However, any such method will naturally run into problems when it encounters a zero-velocity state, and one indeed finds that both the nonlinear coefficient and the dispersion parameter derived in the $z$-propagation schemes diverge at $\beta=0$. A further problem is that modeling of pulse propagation around $\beta=0$ should account for both forward- and backward-propagating states, whose boundary conditions should be specified at opposite ends of the fiber, since physically one controls the input to the fiber, whereas calculation of the output is desired. This further complicates the formulation of a $z$-stepping numerical method. Both of these problems can be circumvented by propagating in the time domain, since time propagation is unidirectional and cannot be slowed down or stopped. The derivation of timepropagated nonlinear equations for forward-propagating states only has been discussed by Kolesik and Moloney [12]. An early study of nonlinear propagation in slow fiber modes utilized a full finite-difference time-domain propagation of Maxwell's equations, and also derived a time-domain NLSE for the slow mode [13]. In this paper, a novel and more general time-propagating formalism is derived, which includes both instantaneous (Kerr) and delayed (Raman) nonlinear response, and can in principle handle both slow and fast states and their interactions. The formalism is applied to slow-state solitons, and it is shown that the Raman effect will act to exponentially decrease their velocity, such that the zero-velocity soliton becomes an attractor state of the system. To the author's knowledge, this aspect of soliton propagation in slow-light states has not previously been discussed in the literature.

\section{FORMAL THEORY}

The starting point is the Maxwell equations in the presence of a nonlinear polarization term:

$$
\begin{gathered}
\nabla \times \mathbf{E}=-\mu_{0} \frac{\partial \mathbf{H}}{\partial t} \\
\nabla \times \mathbf{H}=\varepsilon_{0} \varepsilon\left(\mathbf{r}_{\perp}\right) \frac{\partial \mathbf{E}}{\partial t}+\frac{\partial \mathbf{P}_{\mathrm{NL}}}{\partial t} .
\end{gathered}
$$


Here $\varepsilon$ is the relative dielectric constant of the medium, assumed $z$-independent for a straight waveguide, and $\mathbf{P}_{\mathrm{NL}}$ is the nonlinear part of the induced polarization. The $\mathbf{E}$ and $\mathbf{H}$ fields are expanded into modal fields:

$$
\begin{aligned}
& \mathbf{H}(\mathbf{r}, t)= \frac{1}{\sqrt{2 \pi}} \sum_{m} \int \mathrm{d} \beta\left[\left(A_{m}(t, \beta)\right.\right. \\
&\left.+\delta_{m}(t, \beta)\right) \mathbf{h}_{m}\left(\mathbf{r}_{\perp}, \beta\right) e^{i\left(\omega_{m}(\beta) t-\beta z\right)} \\
&+\left(A_{m}^{*}(t,-\beta)+\delta_{m}^{*}(t,-\beta)\right) \\
&\left.\times \mathbf{h}_{m}^{*}\left(\mathbf{r}_{\perp},-\beta\right) e^{-i\left(\omega_{m}(\beta) t+\beta z\right)}\right] \\
& \mathbf{E}(\mathbf{r}, t)=\frac{1}{\sqrt{2 \pi}} \sum_{m} \int \mathrm{d} \beta\left[A_{m}(t, \beta) \mathbf{e}_{m}\left(\mathbf{r}_{\perp}, \beta\right) e^{i\left(\omega_{m}(\beta) t-\beta z\right)}\right. \\
&\left.+A_{m}^{*}(t,-\beta) \mathbf{e}_{m}^{*}\left(\mathbf{r}_{\perp},-\beta\right) e^{-i\left(\omega_{m}(\beta) t+\beta z\right)}\right]
\end{aligned}
$$

where the $\beta$ integration extends over both positive and negative values. The modes $\mathbf{h}_{m}(\mathbf{r}, t ; \beta), \mathbf{e}_{m}(\mathbf{r}, t ; \beta)$ given by

$$
\begin{aligned}
\mathbf{h}_{m}(\mathbf{r}, t ; \beta) & =\mathbf{h}_{m}\left(\mathbf{r}_{\perp}, \beta\right) e^{i\left(\omega_{m}(\beta) t-\beta z\right)}, \\
\mathbf{e}_{m}(\mathbf{r}, t ; \beta) & =\mathbf{e}_{m}\left(\mathbf{r}_{\perp}, \beta\right) e^{i\left(\omega_{m}(\beta) t-\beta z\right)},
\end{aligned}
$$

fulfill the linear Maxwell equations

$$
\begin{aligned}
& \nabla \times \mathbf{e}_{m}(\mathbf{r}, t ; \beta)=-\mu_{0} \frac{\partial \mathbf{h}_{m}(\mathbf{r}, t ; \beta)}{\partial t}, \\
& \nabla \times \mathbf{h}_{m}(\mathbf{r}, t ; \beta)=\varepsilon_{0} \varepsilon\left(\mathbf{r}_{\perp}\right) \frac{\partial \mathbf{e}_{m}(\mathbf{r}, t ; \beta)}{\partial t},
\end{aligned}
$$

and it is readily shown that the fields $\mathbf{E}$ and $\mathbf{H}$ are real, and that, in the case of linear propagation, the expansion coefficients $A_{m}(t, \beta)$ are time-independent, and the $\delta_{m}(t, \beta)$ coefficients are zero. In the case of nonlinear propagation, the $A_{m}(t, \beta)$ coefficients will acquire a time dependence, and the $\delta_{m}(t, \beta)$ coefficients must then be nonzero, because both $\mathbf{E}, \mathbf{H}$ and $\mathbf{e}, \mathbf{h}$ must satisfy Eq. (1). Inserting Eqs. (3) and (4) into Eq. (1), one finds that this requirement leads to the equation

$$
\frac{\partial A_{m}(t, \beta)}{\partial t}=-i \omega_{m}(\beta) \delta_{m}(t, \beta)-\frac{\partial \delta_{m}(t, \beta)}{\partial t} .
$$

Assuming that $\partial^{2} A_{m}(t, \beta) / \partial t^{2}$ is negligible, this equation is approximately satisfied by

$$
-i \omega_{m}(\beta) \delta_{m}(t, \beta)=\frac{\partial A_{m}(t, \beta)}{\partial t},
$$

an approximation which will be used below to obtain a closedform expression for $\partial A_{m}(t, \beta) / \partial t$. It should be noted that the assumption of slowly varying $A_{m}(t, \beta)$ coefficients is not related to the duration of the pulse but rather to the time scale over which significant spectral changes occur, due to nonlinear processes. In the strictly linear regime, $A_{m}(t, \beta)$ would be constant and $\delta_{m}(t, \beta)=0$ regardless of the pulse duration.
The fields $\mathbf{e}$ and $\mathbf{h}$ are normalized so that

$$
\begin{aligned}
& \varepsilon_{0} \int \mathrm{d} \mathbf{r}_{\perp} \varepsilon\left(\mathbf{r}_{\perp}\right) \mathbf{e}_{m}^{*}\left(\mathbf{r}_{\perp}, \beta\right) \cdot \mathbf{e}_{n}\left(\mathbf{r}_{\perp}, \beta\right) \\
& \quad=\mu_{0} \int \mathrm{d} \mathbf{r}_{\perp} \mathbf{h}_{m}^{*}\left(\mathbf{r}_{\perp}, \beta\right) \cdot \mathbf{h}_{n}\left(\mathbf{r}_{\perp}, \beta\right)=\frac{1}{2} \delta_{m n},
\end{aligned}
$$

which implies that the time-averaged field energy in the pulse is given by

$$
\left\langle\int \mathrm{d} \mathbf{r} \mathbf{E} \cdot \mathbf{D}\right\rangle_{t}=\sum_{m} \int \mathrm{d} \beta\left|A_{m}(t, \beta)\right|^{2} .
$$

The transverse fields $\mathbf{e}_{m}\left(\mathbf{r}_{\perp}, \beta\right)$ and $\mathbf{h}_{m}\left(\mathbf{r}_{\perp}, \beta\right)$ fulfill the condition

$$
\mathbf{e}_{m}^{*}\left(\mathbf{r}_{\perp},-\beta\right)=\mathbf{e}_{m}\left(\mathbf{r}_{\perp}, \beta\right), \quad \mathbf{h}_{m}^{*}\left(\mathbf{r}_{\perp},-\beta\right)=-\mathbf{h}_{m}\left(\mathbf{r}_{\perp}, \beta\right),
$$

as may be seen by taking the complex conjugate of Eq. (ㅁ). From Eq. (2), one obtains

$$
\begin{gathered}
\int \operatorname{dre}_{m}^{*}(\mathbf{r}, t ; \beta) \cdot\left[\frac{\partial \mathbf{P}_{\mathrm{NL}}}{\partial t}+\varepsilon_{0} \varepsilon \frac{\partial \mathbf{E}}{\partial t}\right]=\int \mathrm{d} \mathbf{r} \mathbf{e}_{m}^{*}(\mathbf{r}, t ; \beta) \cdot \nabla \times \mathbf{H} \\
=\int \mathrm{d} \mathbf{r} \mathbf{H} \cdot \nabla \times \mathbf{e}_{m}^{*}(\mathbf{r}, t ; \beta)=i \mu_{0} \omega_{m}(\beta) \int \mathrm{d} \mathbf{r} \mathbf{H} \cdot \mathbf{h}_{m}^{*}(\mathbf{r}, t ; \beta) .
\end{gathered}
$$

Inserting the field expansions Eqs. (3) and (4) and using Eqs. (ㅁ), (9)), and (11) yields the result

$$
\begin{aligned}
& \frac{1}{\sqrt{2 \pi}} \int \mathrm{d} \mathbf{r e}_{m}^{*}(\mathbf{r}, t ; \beta) \cdot \frac{\partial \mathbf{P}_{\mathrm{NL}}}{\partial t}+\left[i \omega_{m}(\beta) A_{m}(t, \beta)+\frac{\partial A_{m}(t, \beta)}{\partial t}\right. \\
& \left.\quad+\left(\frac{\partial A^{*}(t,-\beta)}{\partial t}-i \omega_{m}(\beta) A_{m}^{*}(t,-\beta)\right) e^{-2 i \omega_{m}(\beta) t}\right] \\
& \quad \times \varepsilon_{0} \int \mathrm{d} \mathbf{r}_{\perp} \varepsilon\left(\mathbf{r}_{\perp}\right)\left|\mathbf{e}_{m}\left(\mathbf{r}_{\perp}, \beta\right)\right|^{2} \\
& =i \omega_{m}(\beta) \mu_{0}\left[A_{m}(t, \beta)+\delta_{m}(t, \beta)-\left(A_{m}^{*}(t,-\beta)\right.\right. \\
& \left.\left.\quad+\delta_{m}^{*}(t,-\beta)\right) e^{-2 i \omega_{m}(\beta) t}\right] \int \mathrm{d} \mathbf{r}_{\perp}\left|\mathbf{h}_{m}\left(\mathbf{r}_{\perp}, \beta\right)\right|^{2}, \\
& \Downarrow \frac{\partial A_{m}(t, \beta)}{\partial t}+\frac{\partial A^{*}(t,-\beta)}{\partial t} e^{-2 i \omega_{m}(\beta) t} \\
& =-\frac{1}{\sqrt{2 \pi}} \int \mathrm{d} \mathbf{r} \mathbf{e}_{m}^{*}(\mathbf{r}, t ; \beta) \cdot \frac{\partial \mathbf{P}_{\mathrm{NL}}}{\partial t} .
\end{aligned}
$$

To proceed further, the functional form of the nonlinear polarization must be specified. In this work, a $\mathbf{P}_{\mathrm{NL}}$ consisting of Kerr and Raman terms will be considered [14]:

$$
\begin{aligned}
\mathbf{P}_{\mathrm{NL}}(\mathbf{r}, t)= & \varepsilon_{0}\left[\chi_{K}^{(3)}(\mathbf{r}) \vdots \mathbf{E}(\mathbf{r}, t) \mathbf{E}(\mathbf{r}, t) \mathbf{E}(\mathbf{r}, t)\right. \\
& \left.+\chi_{R}^{(3)}(\mathbf{r}) \vdots \mathbf{E}(\mathbf{r}, t) \int_{-\infty}^{t} \mathrm{~d} t_{1} R\left(t-t_{1}\right) \mathbf{E}\left(\mathbf{r}, t_{1}\right) \mathbf{E}\left(\mathbf{r}, t_{1}\right)\right],
\end{aligned}
$$

where $\chi_{K}^{(3)}$ and $\chi_{R}^{(3)}$ are the Kerr and Raman susceptibility tensors, respectively. If the field expansion (4) is inserted into Eq. (14), and the result is substituted into Eq. (13), the right-hand side will consist of terms oscillating with frequencies $\sim 0, \pm 2 \omega_{0}$, and $-4 \omega_{0}$, where $\omega_{0}$ is some suitably chosen 
center frequency, and the spectral width of the light is assumed smaller than $\omega_{0}$. The $+2 \omega_{0}$ and $-4 \omega_{0}$ terms correspond to third-harmonic generation processes, which are assumed to be negligible here, since the PBG-guided slow mode will not exist at the third-harmonic frequency, and other fast-moving modes will be very far from phase matching. A propagation equation is obtained by equating the slowly varying terms in Eq. (13). First, the part of $\mathbf{P}_{\mathrm{NL}}$ oscillating at $\sim \omega_{0}$, which will hereafter be denoted $\mathbf{P}_{\mathrm{NL}}$, is determined:

$$
\begin{aligned}
\mathbf{P}_{\mathrm{NL}}(\mathbf{r}, t)= & \sum_{n p q} \frac{\varepsilon_{0}}{(2 \pi)^{3 / 2}} \int \mathrm{d} \beta_{1-3} e^{-i\left(\beta_{1}+\beta_{2}+\beta_{3}\right) z}\left\{\left[2 \tilde{A}_{n}\left(t, \beta_{1}\right) \tilde{A}_{p}\left(t, \beta_{2}\right)\right.\right. \\
& \times \tilde{A}_{q}^{*}\left(t,-\beta_{3}\right)+\tilde{A}_{n}^{*}\left(t,-\beta_{1}\right) \tilde{A}_{p}\left(t, \beta_{2}\right) \\
& \left.\times \tilde{A}_{q}\left(t, \beta_{3}\right)\right] \chi_{K}^{(3)}: \mathbf{e}_{n}\left(\mathbf{r}_{\perp}, \beta_{1}\right) \mathbf{e}_{p}\left(\mathbf{r}_{\perp}, \beta_{2}\right) \mathbf{e}_{q}^{*}\left(\mathbf{r}_{\perp},-\beta_{3}\right) \\
& +\left[2 \tilde{A}_{n}\left(t, \beta_{1}\right) \int_{-\infty}^{t} \mathrm{~d} t_{1} R\left(t-t_{1}\right) \tilde{A}_{p}\left(t_{1}, \beta_{2}\right)\right. \\
& \times \tilde{A}_{q}^{*}\left(t_{1},-\beta_{3}\right)+\tilde{A}_{n}^{*}\left(t,-\beta_{1}\right) \int_{-\infty}^{t} \mathrm{~d} t_{1} R\left(t-t_{1}\right) \\
& \left.\times \tilde{A}_{p}\left(t_{1}, \beta_{2}\right) \tilde{A}_{q}\left(t_{1}, \beta_{3}\right)\right] \chi_{R}^{(3)}: \mathbf{e}_{n}\left(\mathbf{r}_{\perp}, \beta_{1}\right) \\
& \left.\mathbf{e}_{p}\left(\mathbf{r}_{\perp}, \beta_{2}\right) \mathbf{e}_{q}^{*}\left(\mathbf{r}_{\perp},-\beta_{3}\right)\right\}
\end{aligned}
$$

$$
\tilde{A}_{m}(t, \beta)=A_{m}(t, \beta) e^{i \omega_{m}(\beta) t}
$$

Next, the time derivative of $\mathbf{P}_{\mathrm{NL}}$ is evaluated. Here it is assumed that $\partial \tilde{A} / \partial t \approx i \omega \tilde{A}$, i.e., that $\partial A / \partial t$ is negligible compared to $i \omega A$. This is justified, since $A$ is a constant in the linear case, so $\partial A / \partial t$ must at least be proportional to $\chi^{(3)}$, and a term proportional to both $\partial A / \partial t$ and $\chi^{(3)}$ would thus be of second order in $\chi^{(3)}$. It will also be assumed that the last Raman term in Eq. (16) is negligible, since the Raman response function is convoluted with a term oscillating at $\sim 2 \omega_{0}[\underline{15}]$. With these approximations, one finds

$$
\begin{aligned}
\frac{\partial \mathbf{P}_{\mathrm{NL}}(\mathbf{r}, t)}{\partial t}= & \sum_{n p q} \frac{\varepsilon_{0}}{(2 \pi)^{3 / 2}} \int \mathrm{d} \beta_{1-3} e^{-i\left(\beta_{1}+\beta_{2}+\beta_{3}\right) z}\left\{i \left[2 \left(\omega_{n}\left(\beta_{1}\right)\right.\right.\right. \\
& \left.+\omega_{p}\left(\beta_{2}\right)-\omega_{q}\left(\beta_{3}\right)\right) \times \tilde{A}_{n}\left(t, \beta_{1}\right) \tilde{A}_{p}\left(t, \beta_{2}\right) \tilde{A}_{q}^{*}\left(t,-\beta_{3}\right) \\
& +\left(\omega_{p}\left(\beta_{2}\right)+\omega_{q}\left(\beta_{3}\right)-\omega_{n}\left(\beta_{1}\right)\right) \tilde{A}_{n}^{*}\left(t,-\beta_{1}\right) \tilde{A}_{p}\left(t, \beta_{2}\right) \\
& \left.\times \tilde{A}_{q}\left(t, \beta_{3}\right)\right] \times \chi_{K}^{(3)}: \mathbf{e}_{n}\left(\mathbf{r}_{\perp}, \beta_{1}\right) \mathbf{e}_{p}\left(\mathbf{r}_{\perp}, \beta_{2}\right) \mathbf{e}_{q}^{*}\left(\mathbf{r}_{\perp},-\beta_{3}\right) \\
& +2 \tilde{A}_{n}\left(t, \beta_{1}\right) \int_{-\infty}^{t} \mathrm{~d} t_{1}\left(i \omega_{n}\left(\beta_{1}\right) R\left(t-t_{1}\right)\right. \\
& \left.+R^{\prime}\left(t-t_{1}\right)\right) \tilde{A}_{p}\left(t_{1}, \beta_{2}\right) \tilde{A}_{q}^{*}\left(t_{1},-\beta_{3}\right) \\
& \left.\times \chi_{R}^{(3)}: \mathbf{e}_{n}\left(\mathbf{r}_{\perp}, \beta_{1}\right) \mathbf{e}_{p}\left(\mathbf{r}_{\perp}, \beta_{2}\right) \mathbf{e}_{q}^{*}\left(\mathbf{r}_{\perp},-\beta_{3}\right)\right\}
\end{aligned}
$$

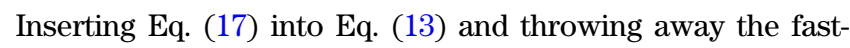
oscillating term on the left-hand side, one obtains the propagation equation

$$
\begin{aligned}
& \frac{\partial A_{m}(t, \beta)}{\partial t}=-e^{-i \omega_{m}(\beta) t} \frac{\varepsilon_{0}}{2 \pi} \sum_{n p q} \int \mathrm{d} \beta_{1} \mathrm{~d} \beta_{2}\left\{K_{m n p q}\left(\beta, \beta_{1}, \beta_{2}\right)\right. \\
& \times\left[2 \tilde{A}_{n}\left(t, \beta_{1}\right) \tilde{A}_{p}\left(t, \beta_{2}\right) \tilde{A}_{q}^{*}\left(t, \beta_{1}+\beta_{2}-\beta\right)\right. \\
& \times i\left(\omega_{n}\left(\beta_{1}\right)+\omega_{p}\left(\beta_{2}\right)-\omega_{q}\left(\beta_{1}+\beta_{2}-\beta\right)\right) \\
&+\tilde{A}_{n}^{*}\left(t,-\beta_{1}\right) \tilde{A}_{p}\left(t, \beta_{2}\right) \tilde{A}_{q}\left(t, \beta-\beta_{1}-\beta_{2}\right) i\left(\omega_{p}\left(\beta_{2}\right)\right. \\
&\left.\left.+\omega_{q}\left(\beta_{1}+\beta_{2}-\beta\right)-\omega_{n}\left(\beta_{1}\right)\right)\right] \\
&+R_{m n p q}\left(\beta, \beta_{1}, \beta_{2}\right) 2 \tilde{A}_{n}\left(t, \beta_{1}\right) \\
& \times \int_{-\infty}^{t} \mathrm{~d} t_{1} \tilde{A}_{p}\left(t_{1}, \beta_{2}\right) \tilde{A}_{q}^{*}\left(t_{1}, \beta_{1}+\beta_{2}-\beta\right)\left(R^{\prime}\left(t-t_{1}\right)\right. \\
&\left.+i \omega_{n}\left(\beta_{1}\right) R\left(t-t_{1}\right)\right), \\
& K_{m n p q}(\left(\beta_{1}, \beta_{2}\right)=\int \mathrm{d} \mathbf{r}_{\perp} \mathbf{e}_{m}^{*}\left(\mathbf{r}_{\perp}, \beta\right) \\
& \cdot \chi_{K}^{(3)}\left(\mathbf{r}_{\perp}\right) \vdots \mathbf{e}_{n}\left(\mathbf{r}_{\perp}, \beta_{1}\right) \mathbf{e}_{p}\left(\mathbf{r}_{\perp}, \beta_{2}\right) \mathbf{e}_{q}^{*}\left(\mathbf{r}_{\perp}, \beta_{1}+\beta_{2}-\beta\right), \\
& R_{m n p q}\left(\beta, \beta_{1}, \beta_{2}\right)=\int \mathrm{d} \mathbf{r}_{\perp} \mathbf{e}_{m}^{*}\left(\mathbf{r}_{\perp}, \beta\right) \\
& \cdot \chi_{R}^{(3)}\left(\mathbf{r}_{\perp}\right) \vdots \mathbf{e}_{n}\left(\mathbf{r}_{\perp}, \beta_{1}\right) \mathbf{e}_{p}\left(\mathbf{r}_{\perp}, \beta_{2}\right) \mathbf{e}_{q}^{*}\left(\mathbf{r}_{\perp}, \beta_{1}+\beta_{2}-\beta\right) .
\end{aligned}
$$

Equation (18) is fairly general. In the following, it will be simplified to treat the case of a pulse propagating in a single optical mode. Thus, the mode indices on frequencies and $A$ coefficients will from now on be suppressed. Furthermore, the complicated integration kernels $K$ and $R$ will be simplified to obtain a numerically tractable propagation equation [11]. In this work, the mode profile dispersion will be neglected, so that all $\beta$-dependent transverse fields are replaced by the $\beta=0$ field distribution, and $K, R$ become constant functions. Since the transverse fields may be taken real at $\beta=0$, the $\chi^{(3)}$ tensor products may be written as

$$
\begin{aligned}
& K=\chi_{K x x x x}^{(3)} \int_{m} \mathrm{~d} \mathbf{r}_{\perp}\left|\mathbf{e}\left(\mathbf{r}_{\perp}, 0\right)\right|^{4}, \\
& R=\chi_{R x x x x}^{(3)} \int_{m} \mathrm{~d} \mathbf{r}_{\perp}\left|\mathbf{e}\left(\mathbf{r}_{\perp}, 0\right)\right|^{4},
\end{aligned}
$$

where it has been assumed that the microstructured fiber cross section is composed of alternating sections of dielectric material and air and that only the dielectric material contributes appreciably to the nonlinear effects. Therefore, the cross-sectional integral has been restricted to the region where dielectric material is present. That only the $x x x x$ component of $\chi^{(3)}$ matters may be seen by introducing a local coordinate system at each point in the transverse plane whose $x$-axis is aligned with the field polarization vector at that point. The expression for $K$ can be further rewritten as

$$
\begin{aligned}
K & =\chi_{K x x x x}^{(3)} \frac{\int_{m} \mathrm{~d} \mathbf{r}_{\perp} \varepsilon^{2}\left(\mathbf{r}_{\perp}\right)\left|\mathbf{e}\left(\mathbf{r}_{\perp}, 0\right)\right|^{4}}{4 \varepsilon_{0}^{2} \varepsilon_{m}^{2}\left[\int \mathrm{d} \mathbf{r}_{\perp} \varepsilon\left(\mathbf{r}_{\perp}\right)\left|\mathbf{e}\left(\mathbf{r}_{\perp}, 0\right)\right|^{2}\right]^{2}} \\
& \equiv \frac{\chi_{K x x x x}^{(3)}}{4 \varepsilon_{0}^{2} \varepsilon_{m}^{2} A_{\text {eff }}(\beta=0)},
\end{aligned}
$$

with a similar expression for $R$. Here $\varepsilon_{m}$ is the relative permittivity of the nonlinear material. 
The effective-area parameter $A_{\text {eff }}$ defined in Eq. (22) differs from those usually obtained in $z$-propagating formulations. One expression that can be simply related to $A_{\text {eff }}$ was suggested in Ref. [16]:

$$
\begin{aligned}
\tilde{A}_{\mathrm{eff}}(\beta) & =\varepsilon_{m}\left(\frac{v_{g}}{c}\right)^{2} \frac{\left[\int \mathrm{d} \mathbf{r}_{\perp} \varepsilon\left(\mathbf{r}_{\perp}\right)\left|\mathbf{e}\left(\mathbf{r}_{\perp}, \beta\right)\right|^{2}\right]^{2}}{\int_{m} \mathrm{~d} \mathbf{r}_{\perp} \varepsilon^{2}\left(\mathbf{r}_{\perp}\right)\left|\mathbf{e}\left(\mathbf{r}_{\perp}, \beta\right)\right|^{4}} \\
& =\varepsilon_{m}\left(\frac{v_{g}}{c}\right)^{2} A_{\text {eff }}(\beta),
\end{aligned}
$$

where $v_{g}$ is the group velocity. This effective area vanishes for a zero-velocity state, whereas $A_{\text {eff }}$ remains finite. The difference is due to the fact that the transverse fields in $z$-propagating schemes are normalized to the $z$-propagating power, whereas the fields in the time-propagating formalism developed here are normalized to the energy density, as shown by Eq. (9). An alternative formulation has recently been proposed by Afshar V. and Monro [7], in which both an effective-area parameter and an averaged nonlinear refractive index is determined from the modal fields and fiber cross section. In this formulation, the proposed effective area would stay finite for $\beta \rightarrow 0$; however, the averaged nonlinear index would diverge, again due to the vanishing of the power normalization integral.

With the above approximations to $K$ and $R$ and the further definitions

$$
\begin{aligned}
& B(t, \beta)=i \omega(\beta) \tilde{A}_{m}(t, \beta), \\
& \tilde{A}(t, z)=\frac{1}{\sqrt{2 \pi}} \int \mathrm{d} \beta e^{-i \beta z} \tilde{A}_{m}(t, \beta), \\
& B(t, z)=\frac{1}{\sqrt{2 \pi}} \int \mathrm{d} \beta e^{-i \beta z} B(t, \beta),
\end{aligned}
$$

the propagation equation can be rewritten as

$$
\begin{aligned}
& \frac{\partial A(t, \beta)}{\partial t}=-e^{-i \omega(\beta) t} \frac{N_{2}}{A_{\text {eff }}} \frac{1}{\sqrt{2 \pi}} \int \mathrm{d} z e^{i \beta z}\left[( 1 - f _ { R } ) \left(2|\tilde{A}(t, z)|^{2} B(t, z)\right.\right. \\
& \left.+\tilde{A}^{2}(t, \boldsymbol{z}) B^{*}(t, \boldsymbol{z})\right)+f_{R}(\tilde{A}(t, \boldsymbol{z}) G(t, \boldsymbol{z}) \\
& +B(t, z) F(t, z))] \text {, } \\
& F(t, z)=\int_{-\infty}^{t} \mathrm{~d} t_{1} R\left(t-t_{1}\right)\left|\tilde{A}\left(t_{1}, z\right)\right|^{2}, \\
& G(t, z)=\int_{-\infty}^{t} \mathrm{~d} t_{1} R^{\prime}\left(t-t_{1}\right)\left|\tilde{A}\left(t_{1}, z\right)\right|^{2}, \\
& N_{2}=\frac{3 \chi_{s}^{(3)}}{4 \varepsilon_{0} \varepsilon_{m}^{2}}, \quad \chi_{s}^{(3)}=\chi_{K x x x x}^{(3)}+\frac{2}{3} \chi_{R x x x x}^{(3)}, \quad f_{R}=\frac{2 \chi_{R x x x x}^{(3)}}{3 \chi_{s}^{(3)}}
\end{aligned}
$$

These are the propagation equations, which will be solved numerically. Approximating the Raman term as an instantaneous interaction, assuming $B \approx i \omega_{0} \tilde{A}$, and recasting Eq. (25) as an equation for $\tilde{A}$, one obtains

$$
\begin{aligned}
\frac{\partial \tilde{A}_{m}(t, \beta)}{\partial t}= & i \omega(\beta) \tilde{A}_{m}(t, \beta) \\
& -i \omega_{0} \frac{N_{2}}{A_{\text {eff }}} \frac{1}{\sqrt{2 \pi}} \int \mathrm{d} z e^{i \beta z} \tilde{A}(t, z)|\tilde{A}(t, z)|^{2} .
\end{aligned}
$$

Inserting $\omega(\beta)=\omega_{0}+\left(\omega_{2} / 2\right) \beta^{2}$ and transforming the equation into the $z$ domain, this becomes the well-known NLSE

$$
\frac{\partial \tilde{A}(t, z)}{\partial t}=i\left[\omega_{0}-\frac{\omega_{2}}{2} \frac{\partial^{2}}{\partial z^{2}}\right] \tilde{A}(t, z)-i \omega_{0} \frac{N_{2}}{A_{\mathrm{eff}}} \tilde{A}(t, z)|\tilde{A}(t, z)|^{2}
$$

The $i \omega_{0}$ term can be eliminated by letting $\tilde{A}(t, z) \rightarrow \tilde{A}(t, z) e^{i \omega_{0} t}$. This propagation equation was derived from first-order perturbation theory by Soljačić et al. [13]. In the present derivation, it appears as a limiting case of the more general Eq. (25), which is not significantly more challenging from a numerical point of view.

Whereas dispersion in the NLSE is quantified by the parameter $\beta_{2}=d^{2} \beta / d \omega^{2}$, in Eq. (29) it is given by $\omega_{2}=d^{2} \omega / d \beta^{2}$. If $\omega_{2}$ is positive, as is typically the case, Eq. (29) is equivalent to the NLSE in the case of anomalous dispersion, $\beta_{2}<0$. This implies the existence of solitonic solutions of the form

$$
\begin{aligned}
& A(t, z)=\sqrt{\xi_{0}} \operatorname{sech}\left(\frac{z-v_{g} t}{z_{0}}\right) e^{i t\left(\omega\left(\beta_{0}\right)-1 / 2 T_{\mathrm{NL}}\right)} e^{-i \beta_{0} z}, \\
& z_{0}^{2}=\frac{\omega_{2}}{\Gamma \xi_{0}}, \quad \Gamma=\frac{\omega_{0} N_{2}}{A_{\mathrm{eff}}}, \quad T_{\mathrm{NL}}=\frac{1}{\Gamma \xi_{0}},
\end{aligned}
$$

where $\xi_{0}$ is the peak energy density of the soliton, and $v_{g}$ is the group velocity at $\beta_{0}$. This formula describes both stationary and moving solitons. Sufficiently energetic pulses of any shape and duration can be expected to break up into solitons, if coupled into a slow mode of the fiber.

The inclusion of the Raman term breaks the equivalence between the $t$ - and $z$-propagating nonlinear equations, which is to be expected because the Raman interaction is nonlocal in time but not in space. Evaluation of the functions $F$ and $G$ is numerically cumbersome in the general case, because an integral over $A$ functions from many previous time steps must be performed. However, if the Raman response function is approximated by a single damped-harmonic oscillator form [14],

$$
R(t)=\frac{\tau_{1}^{2}+\tau_{2}^{2}}{\tau_{1} \tau_{2}^{2}} \sin \left(\frac{t}{\tau_{1}}\right) e^{-\frac{t}{\tau_{2}}},
$$

one may write

$$
\begin{aligned}
G(t, \boldsymbol{z})= & \frac{\tau_{1}^{2}+\tau_{2}^{2}}{2 i \tau_{1} \tau_{2}^{2}} \int_{-\infty}^{t} \mathrm{~d} t_{1}\left[\left(\frac{i}{\tau_{1}}-\frac{1}{\tau_{2}}\right) e^{\left(\frac{i}{\tau_{1}}-\frac{1}{\tau_{2}}\right)\left(t-t_{1}\right)}\right. \\
& \left.+\left(\frac{i}{\tau_{1}}+\frac{1}{\tau_{2}}\right) e^{-\left(\frac{i}{\tau_{1}}+\frac{1}{\tau_{2}}\right)\left(t-t_{1}\right)}\right] \times\left|\tilde{A}\left(t_{1}, \boldsymbol{z}\right)\right|^{2} \\
\equiv & G_{+}(t, \boldsymbol{z})+G_{-}(t, \boldsymbol{z}),
\end{aligned}
$$

$$
\frac{\partial G_{ \pm}(t, z)}{\partial t}=\frac{\tau_{1}^{2}+\tau_{2}^{2}}{2 i \tau_{1} \tau_{2}^{2}}\left(\frac{i}{\tau_{1}} \mp \frac{1}{\tau_{2}}\right)|\tilde{A}(t, z)|^{2}+\left( \pm \frac{i}{\tau_{1}}-\frac{1}{\tau_{2}}\right) G_{ \pm}(t, \boldsymbol{z}),
$$




$$
\frac{\partial F(t, z)}{\partial t}=G(t, z) .
$$

Using these formulae, the $F$ and $G$ functions may be updated "on the fly," without referencing earlier values of $\tilde{A}(t, z)$.

\section{NUMERICAL RESULTS}

\section{A. Slow Modes in $\mathrm{As}_{2} \mathrm{Se}_{3}$ Fibers}

To obtain physically reasonable parameters for the nonlinear propagation equation derived in Section 2 , some calculations of mode profiles in high-index $\mathrm{As}_{2} \mathrm{Se}_{3}$ microstructured fibers were undertaken. The linear Maxwell equations for the guided modes were solved by expanding the magnetic field and the dielectric function in-plane waves, using a freely available software package [17]. A supercell of $8 \times 8$ elementary unit cells of the cladding structure was used to incorporate the defect, and a coupling-reducing $\mathbf{k}$ point was used to enhance convergence with respect to supercell size [18]. The resolution of the Fourier grid was $64 \times 64$ points in each cladding unit cell. Oskooi $e t$ al. have earlier studied the formation of in-plane bandgaps and slow-mode guidance in $\mathrm{As}_{2} \mathrm{Se}_{3}$ microstructures at high air-filling fractions and suggested to increase the bandgap size by fabricating a cladding structure consisting of cylinders joined by planar slabs [3] . Unfortunately, such a structure is not the easiest one to fabricate as it does not conform to the "natural" glass flow during a fiber draw. In this paper, the cladding will be described using the geometric model put forward by Mortensen and Nielsen [19]. The structure is shown in Fig. 1 and is characterized by four parameters: the center-to-center airhole distance, or pitch $\Lambda$, the curvature radius of the cladding airhole corners, $d_{\mathrm{cl}}$, the smallest glass bridge width, $w_{b}$, and the curvature radius of the airhole surfaces pointing towards the core, $d_{c}$. A range of cladding structures were investigated by varying $d_{\mathrm{cl}}$ and $w_{b}$; however, the largest bandgaps were found for airholes with $d_{\mathrm{cl}}$ values close to $\Lambda-w_{b}$, having a close to circular shape. For the further simulations, a structure with $d_{\mathrm{cl}}=$ $0.82 \Lambda$ and $w_{b}=0.1 \Lambda$ was chosen, which featured an in-plane bandgap of more than $4 \%$ relative to the center frequency. Two core structures were investigated, one with $d_{c}=d_{\mathrm{cl}}$ and another with $d_{c}=0.5 \Lambda$. In Fig. $\underline{2}$, the bandgaps and guided modes are plotted for the two structures. $\Lambda$ was set to $1 \mu \mathrm{m}$, which gives guided modes at around $2 \mu \mathrm{m}$ for a material refractive index of 2.817. Experimentally, this is an interesting wavelength, since powerful thulium-based fiber laser sources are currently being developed, while at the same time the twophoton absorption, which is problematic at $1.55 \mu \mathrm{m}$, should be significantly suppressed.

At the $\beta=0$ point, all modes can be characterized as being either transverse electric (TE) or transverse magnetic (TM). At finite values of $\beta$, the modes can be divided into nondegenerate modes of either TE or TM character, or doubly degenerate hybrid modes. In the large core, $d_{c}=d_{\mathrm{cl}}$, two nondegenerate modes, one of TE and one of TM character, are found. The TE mode is much less dispersive than the TM mode, and the two modes cross in frequency around $\beta=1.4 \mu \mathrm{m}^{-1}$. In theory, this crossing may appear undisturbed due to the different symmetries of the eigenmodes. In practice, small deviations from the perfectly symmetric fiber structure would likely couple the two modes, leading to an avoided crossing at this point. In the small core, a doubly degenerate

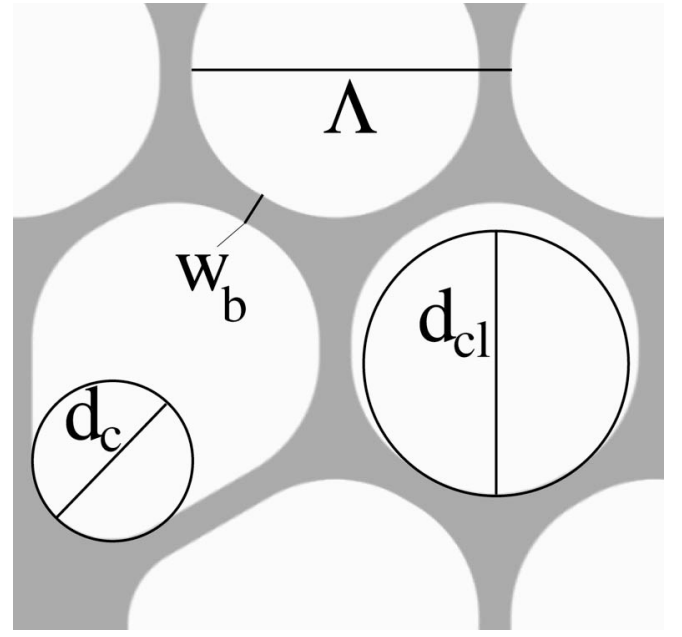

Fig. 1. Generic fiber structure investigated in this work. The defining parameters are the pitch, $\Lambda$, the core and cladding airhole curvature radii, $d_{c}, d_{\mathrm{cl}}$, and the minimal glass bridge width, $w_{b}$. The core center is at the lower left corner of the figure.

hybrid mode, having TE character at $\beta=0$, is guided well inside the bandgap over a large range, whereas two nondegenerate modes are weakly guided at either low or high $\beta$ values. The hybrid mode was chosen to provide input parameters for the nonlinear simulations, as it seems to have fairly representative values of dispersion and effective area. The $\omega_{2}$ parameter was estimated to be $26.23 \mu \mathrm{m}^{2} / \mathrm{ps}$, and the effective area at $\beta=0$ was approximately $1.7 \mu \mathrm{m}^{2}$. The dashed curve in Fig. 2(b) shows the parabolic approximation to the dispersion curve with this value of $\omega_{2}$. A close resemblance is
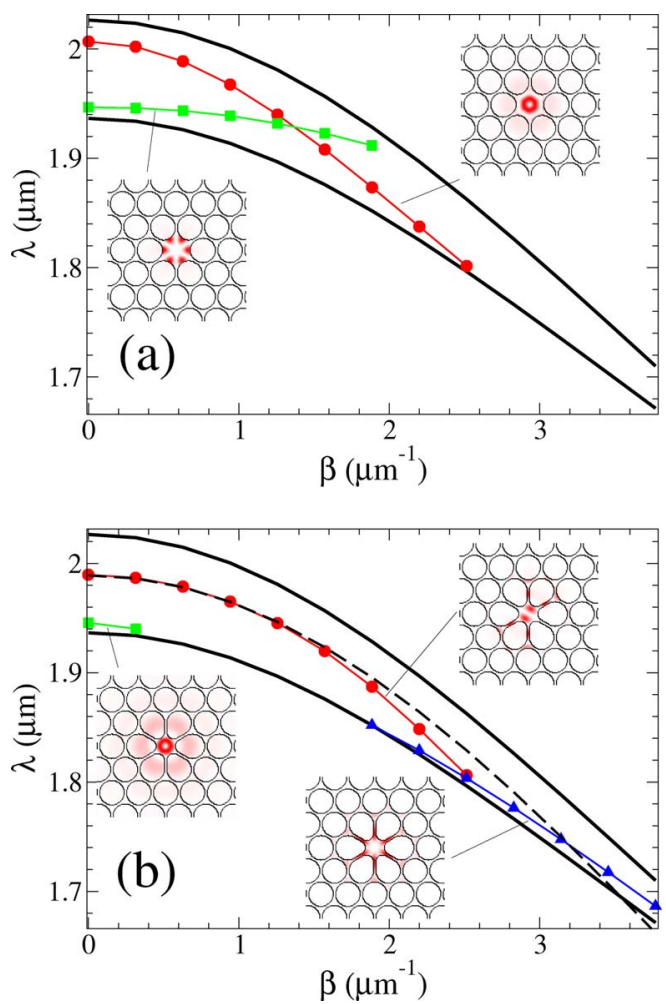

Fig. 2. (Color online) Bandgap boundaries (solid black curves) and guided-mode dispersion curves for two different core designs with (a) $d_{c}=0.82$ and (b) $d_{c}=0.5$. Insets show modal field energy distributions. The dashed black curve in (b) is a quadratic approximation to the hybrid-mode dispersion curve (red circles). 
observed up to $\beta$ values around $1 \mu \mathrm{m}^{-1}$, after which higherorder corrections begin to make a significant difference. In the simulations, pulses peaking at $\beta$ values below $1 \mu \mathrm{m}^{-1}$ will therefore be studied.

If the effective-area parameter defined in Eq. (22) is calculated for both zero and finite $\beta$, a variation of $\sim 20 \%$ is found over the $\beta$ range from $0-1.2 \mu \mathrm{m}^{-1}$, which is relevant for the pulse propagation simulations discussed in Subsection 3.B. Thus, the neglect of mode profile dispersion appears to be a reasonable first approximation, but is at the same time the most significant simplification of the propagation equations used in the this paper. It is emphasized that the inclusion of a $\beta$-dependent effective area in Eq. (25) will not necessarily lead to a correct treatment of mode profile dispersion, since such a procedure would not capture the full complexity of the integration kernels $K$ and $R$ [11].

\section{B. Soliton Propagation}

To numerically solve Eqs. (25) and (26), a fourth-order Runge-Kutta algorithm is used [20]. The functions $\tilde{A}(t, z)$, $B(t, z), F(t, z)$, and $G(t, z)$ are represented on a linear grid with 32,768 points and a discretization step $\Delta z=0.5 \mu \mathrm{m}$. Transformations between the $z$ and $\beta$ representations are done by fast Fourier transforms. The nonlinear refractive index of $\mathrm{As}_{2} \mathrm{Se}_{3}$ was recently measured to vary between $0.75 \times 10^{-5} \mu \mathrm{m}^{2} / \mathrm{W}$ and $1.1 \times 10^{-5} \mu \mathrm{m}^{2} / \mathrm{W}$ at wavelengths from 1470 to $1560 \mathrm{~nm}$ [21]. Here, an estimate of $6 \times 10^{-6} \mu \mathrm{m}^{2} / \mathrm{W}$ is assumed for the longer wavelength of $2 \mu \mathrm{m}$. From this $n_{2}$ value, the value of $\chi^{(3)}$, and thereby $N_{2}$, can be calculated. For the Raman response function, Eq. (31), the peak Raman gain has been estimated to lie around a frequency shift of $7 \mathrm{THz}$ with a spectral width of $\sim 1.4 \mathrm{THz}$ [21]. To match these values, time constants of $\tau_{1}=23 \mathrm{fs}, \tau_{2}=230$ fs were chosen, while $f_{R}$ was set to 0.2 , similar to what one has for silica.

Intuitively, since the Raman scattering process acts to downshift the frequency of the light, it can be expected to push moving solitons towards the zero-velocity soliton state at $\beta=0$, where the frequency is at a minimum. To test this hypothesis, ideal solitons were launched at $\beta=0.6 \mu \mathrm{m}^{-1}$, corresponding to an initial group velocity of $15.7 \mu \mathrm{m} / \mathrm{ps} \approx 0.052 c$. In Fig. 3, the group velocity as a function of propagation time is plotted for three different soliton energies, corresponding to spatial FWHM values of 7.1, 5.8, and $5.0 \mu \mathrm{m}$. The curves were obtained by direct numerical differentiation of the peak pulse position and correspond well to the group velocity at the peak of the spectral function as a function of time. One observes a roughly exponential decay of the soliton velocity, with a decay constant proportional to $E_{p}^{4}$, or equivalently, $z_{0}^{-4}$.

These findings may be explained by treating the Raman interaction as a perturbation to the fundamental soliton, Eq. (30). Considering the averaged $\beta$ value of the pulse

$$
\bar{\beta}=\frac{\int \mathrm{d} \beta|A(t, \beta)|^{2} \beta}{\int \mathrm{d} \beta|A(t, \beta)|^{2}},
$$

the time derivative of this function is given by

$$
\begin{aligned}
\frac{d \bar{\beta}}{d t}= & \frac{\int \mathrm{d} \beta\left[A^{*}(t, \beta) \frac{\partial A(t, \beta)}{\partial t}+A(t, \beta) \frac{\partial A^{*}(t, \beta)}{\partial t}\right] \beta}{\int \mathrm{d} \beta|A(t, \beta)|^{2}} \\
& -\bar{\beta} \frac{\int \mathrm{d} \beta\left[A^{*}(t, \beta) \frac{\partial A(t, \beta)}{\partial t}+A(t, \beta) \frac{\partial A^{*}(t, \beta)}{\partial t}\right]}{\int \mathrm{d} \beta|A(t, \beta)|^{2}} \\
= & \frac{\int \mathrm{d} \beta\left[A^{*}(t, \beta) \frac{\partial A(t, \beta)}{\partial t}+A(t, \beta) \frac{\partial A^{*}(t, \beta)}{\partial t}\right](\beta-\bar{\beta})}{E_{p}} .
\end{aligned}
$$

The time derivative of $A(t, \beta)$ is given by Eq. (18). The Kerr term in the nonlinear polarization does not contribute to the frequency shift, and neither does the Raman term involving $G(t, z)$. However, the Raman term proportional to $F(t, z)$ gives a finite contribution to $d \bar{\beta} / d t$. Inserting the soliton functional form from Eq. (30) into Eq. (26) and expanding the time dependence of $\tilde{A}(t, z)$ to linear order yields

$$
\begin{aligned}
F(t, z)= & \int_{-\infty}^{t} \mathrm{~d} t_{1} R\left(t-t_{1}\right)\left|\tilde{A}\left(t_{1}, \boldsymbol{z}\right)\right|^{2} \\
\approx & |\tilde{A}(t, \boldsymbol{z})|^{2}-\frac{2 \tau_{1}}{\left(\frac{\tau_{1}}{\tau_{2}}+\frac{\tau_{2}}{\tau_{1}}\right)} \frac{\partial|A(t, z)|^{2}}{\partial t} \\
= & |\tilde{A}(t, \boldsymbol{z})|^{2} \\
& -4 \frac{\xi_{0} v_{g}}{z_{0}} \operatorname{sech}^{3}\left(\frac{z-v_{g} t}{z_{0}}\right) \sinh \left(\frac{z-v_{g} t}{z_{0}}\right) \frac{\tau_{1}}{\left(\frac{\tau_{1}}{\tau_{2}}+\frac{\tau_{2}}{\tau_{1}}\right)} .
\end{aligned}
$$

This approximation becomes increasingly well justified as the soliton velocity decreases. Only the term proportional to $\partial \tilde{A} / \partial t$ contributes to $d \bar{\beta} / d t$. Substituting only this part of the $F$ term from Eq. (25) for $\partial A / \partial t$, one finds

$$
\begin{aligned}
A^{*}(t, \beta) \frac{\partial A(t, \beta)}{\partial t}= & -\frac{4 \omega \tau_{1}}{\left(\frac{\tau_{1}}{\tau_{2}}+\frac{\tau_{2}}{\tau_{1}}\right)} z_{0} \xi_{0}^{2} f_{R} \frac{N_{2}}{A_{\mathrm{eff}}} v_{g} \operatorname{sech}\left(\frac{\pi(\beta-\bar{\beta}) z_{0}}{2}\right) \\
& \times \int_{0}^{\infty} \mathrm{d} z \sin (\beta z) \operatorname{sech}^{4}\left(\frac{z-v_{g} t}{z_{0}}\right) \sinh \left(\frac{z-v_{g} t}{z_{0}}\right),
\end{aligned}
$$

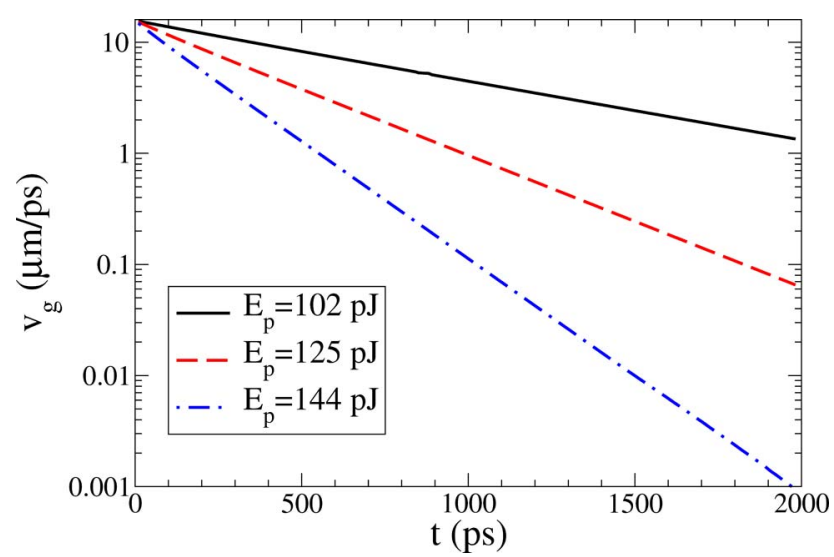

Fig. 3. (Color online) Soliton velocity versus time for three different pulse energies. The curves were determined by direct numerical differentiation of the soliton peak position as a function of time. 
and thereby, using Eq. (36) and the fact that $E_{p}=2 \xi_{0} z_{0}$,

$$
\begin{aligned}
\frac{d \bar{\beta}}{d t}= & -\frac{4 \omega \tau_{1}}{\left(\frac{\tau_{1}}{\tau_{2}}+\frac{\tau_{2}}{\tau_{1}}\right)} \frac{\xi_{0}}{z_{0}^{2}} f_{R} \frac{N_{2}}{A_{\mathrm{eff}}} v_{g} \int_{-\infty}^{\infty} \mathrm{d} u u \operatorname{sech}\left(\frac{\pi u}{2}\right) \\
& \times \int_{0}^{\infty} \mathrm{d} v \sin (u v) \operatorname{sech}^{4}(v) \sinh (v) \\
= & -\frac{16}{15} \frac{\omega \tau_{1}}{\left(\frac{\tau_{1}}{\tau_{2}}+\frac{\tau_{2}}{\tau_{1}}\right)} \frac{\xi_{0}}{z_{0}^{2}} f_{R} \frac{N_{2}}{A_{\text {eff }}} v_{g}
\end{aligned}
$$

Since $v_{g}=\omega_{2} \bar{\beta}$, Eq. (모) implies

$$
v_{g}(t)=v_{g}(t=0) e^{-t / t_{0}}, \quad t_{0}=\frac{15}{16} \frac{\left(\frac{\tau_{1}}{\tau_{2}}+\frac{\tau_{2}}{\tau_{1}}\right)}{\omega \tau_{1}} \frac{z_{0}^{2}}{\xi_{0}} \frac{A_{\text {eff }}}{f_{R} N_{2} \omega_{2}} .
$$

The numerically calculated deceleration rates match very well with this result, especially as the velocity becomes low so that the linear expansion of the temporal pulse profile becomes increasingly accurate. The soliton will propagate no further than

$$
z_{\infty}=\int_{0}^{\infty} \mathrm{d} t v_{g}(t)=v_{g}(t=0) t_{0} .
$$

For an initial group velocity of $15.7 \mu \mathrm{m} / \mathrm{ps}$, this yields $z_{\infty} \approx$ $3.15 \mathrm{~mm}$ for the $144 \mathrm{pJ}$ soliton, which is also in fairly good agreement with the result of the numerical simulations. Note also that the $z_{0}^{2} / \xi_{0}$ dependence of $t_{0}$ may be rewritten as a $z_{0}^{4}$ or $\xi_{0}^{-2}$ dependence using Eq. (30).

A further point of interest is what will happen if two identical solitons are launched with some delay in between, e.g., from a pulsed laser with a fast repetition rate. Since both solitons in isolation would come to rest at the same point in the fiber, some interaction must eventually occur, even if the solitons are initially well separated. From the established theory of solitons, it is known that solitons propagating in close proximity will either attract or repel each other, depending on their relative phase. For most values of the relative phase, the interaction is repulsive. In the case of slow solitons, a similar behavior is found. In Fig. 4, the interaction of two identical solitons, launched with a time interval of 2 ns, is shown for three different values of the relative phase at input. The typical case is that the solitons repel each other with little exchange of energy and end up with a separation of a few tens of micrometers. However, with a carefully chosen relative phase, a strong collision with some transfer of energy is seen.

\section{DISCUSSION}

The results of Section 3 indicate that very slow moving solitons may be obtained in high-index photonic crystal fibers (PCFs) by using the inherent effects of Raman scattering in the glass. In this section, a short discussion regarding the practical feasibility of observing such decelerated solitons will be attempted.

The calculations presented in Section $\underline{3}$ do not account for losses in the fiber. In fact, both waveguiding ("leakage") loss and material absorption/scattering losses will be present. While the leakage loss can always be brought down by enlarging the size of the PBG cladding region, the materialrelated losses are a fundamental limitation. Typical material loss figures cited for fast-moving light in bulk $\mathrm{As}_{2} \mathrm{Se}_{3}$ glass are of the order of $1 \mathrm{~dB} / \mathrm{m}$, with losses for high-quality fibers attaining similar values [4]. When the group velocity approaches zero, the loss per unit length will of course diverge; however, for the time propagation formalism adopted here, the relevant quantity is the loss per unit time, which should remain finite. Assuming a group velocity of $\sim 100 \mu \mathrm{m} / \mathrm{ps}$ in an ordinary fiber mode, or in bulk $\mathrm{As}_{2} \mathrm{Se}_{3}$, a loss of $1 \mathrm{~dB} / \mathrm{m}$ translates into $0.1 \mathrm{~dB} / \mathrm{ns}$, which would not greatly affect the simulations performed here. Another possible limitation
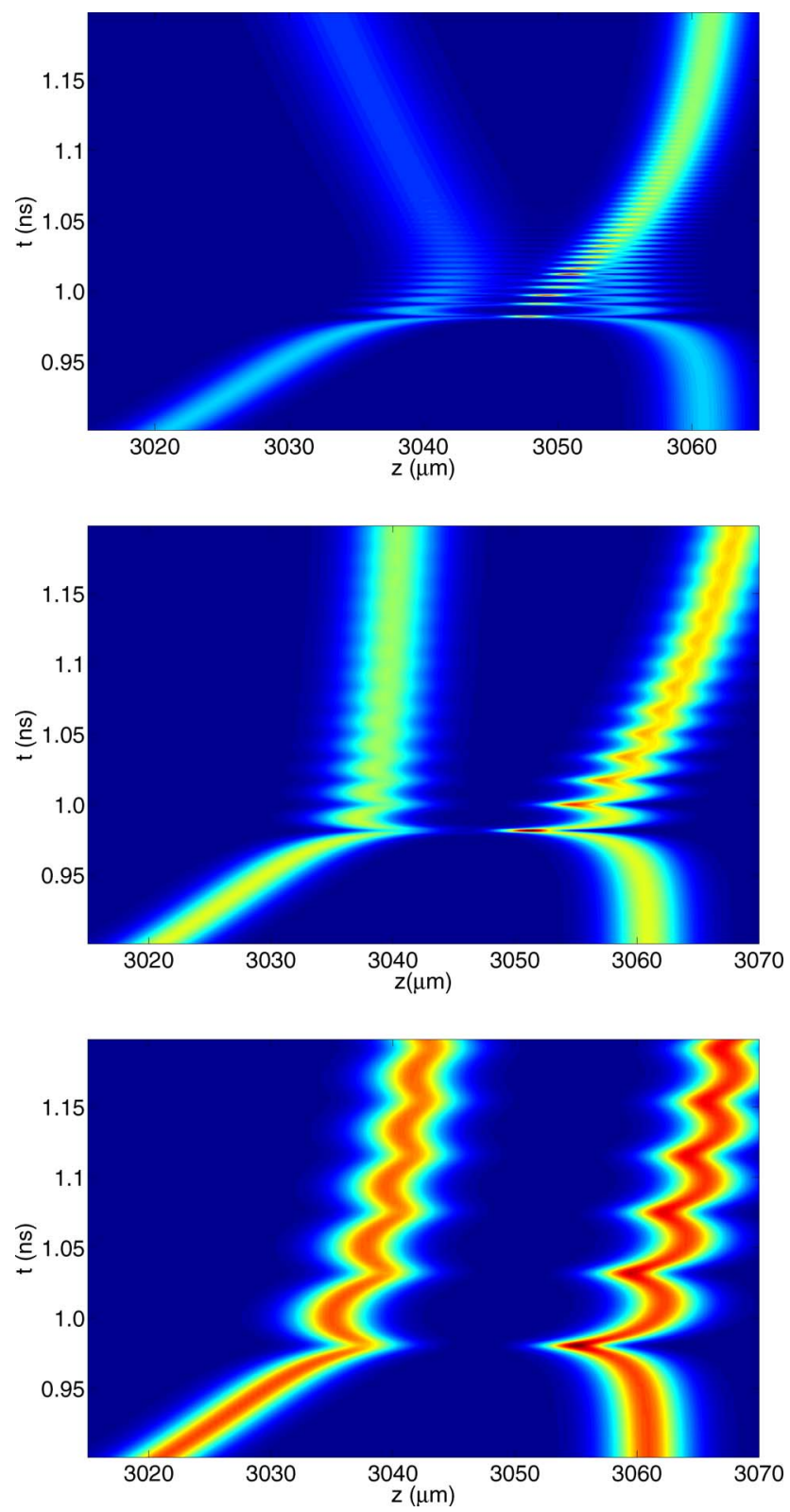

Fig. 4. (Color online) Collisions between $144 \mathrm{pJ}$ solitons launched with a time interval of 2 ns. The time is measured relative to the launch of the second soliton. For a relative input phase $\phi=0.87$ radians (upper left panel), a strong interaction is observed, and the first soliton eventually carries away about $63 \%$ of the total energy. For $\phi=0.9$ radians (upper right), the interaction is already significantly reduced, and the first soliton eventually carries $53 \%$ of the energy. For $\phi=1.0$ radians (lower panel), the interaction is still further reduced, although notable oscillations are seen after the collision. 
may be the breakdown threshold of $\mathrm{As}_{2} \mathrm{Se}_{3}$. The peak energy density of the shortest soliton simulated above is about $25 \mathrm{pJ} / \mu \mathrm{m}$, which in a pulse moving at $100 \mu \mathrm{m} / \mathrm{ps}$ would correspond to a power of $2.5 \mathrm{~kW}$. While the spatial FWHM of the soliton is only about $5 \mu \mathrm{m}$, its temporal duration becomes very long as the velocity decreases. It is then a question whether this energy density can be sustained in a core of only $1.7 \mu \mathrm{m}^{2}$. If the pulse energy is reduced, the Raman deceleration process takes more time and losses may then start to play a role, further reducing the nonlinear effects. A possible remedy may be to amplify the pulse continuously by Raman interaction with a $\mathrm{CW}$ pump in the fundamental (fast) core mode of the fiber.

\section{CONCLUSION}

In conclusion, a time-propagating generalized NLSE has been derived and applied to the case of a slow mode in a high-index PCF. The fiber is found to support stationary as well as moving solitons, and it is shown that Raman scattering will drive the moving solitons exponentially toward the stationary state. If a second soliton is launched, it will interact repulsively with the first soliton for most values of the relative phase between the solitons. Thus, the fundamental zero-velocity soliton appears to be an attractor state in this type of fiber and should be observable if sufficient energy can be coupled into the slow fiber mode by any means.

The general formalism developed in this paper, as well as the fundamental results on soliton behavior in the slow state, will be of high importance for further studies of the nonlinear properties of slow modes in fibers. As for practical applications of the nonlinear soliton deceleration, highly tunable delay lines and light buffering appear to be the most obvious possibilities.

\section{ACKNOWLEDGMENTS}

The author wishes to acknowledge Ole Bang for stimulating discussions and a critical reading of the initial manuscript. Financial support from the Danish National Advanced Technology Foundation is gratefully acknowledged.

\section{REFERENCES}

1. T. Baba, "Slow light in photonic crystals," Nat. Photon. 2, 465-473 (2008).

2. M. Ibanescu, S. G. Johnson, D. Roundy, C. Luo, Y. Fink, and J. D. Joannopoulos, "Anomalous dispersion relations by symmetry breaking in axially uniform waveguides," Phys. Rev. Lett. 92 063903 (2004).

3. A. F. Oskooi, J. D. Joannopoulos, and S. G. Johnson, "Zerogroup-velocity modes in chalcogenide holey photonic-crystal fibers," Opt. Express 17, 10082-10090 (2009).
4. Q. Coulombier, L. Brilland, P. Houizot, T. Chartier, T. N. N'Guyen, F. Smektala, G. Renversez, A. Monteville, D. Méchin, T. Pain, H. Orain, J.-C. Sangleboeuf, and J. Trolès, "Casting method for producing low-loss chalcogenide microstructured optical fibers," Opt. Express 18, 9107-9112 (2010).

5. J. Grgić, S. Xiao, J. Mørk, A.-P. Jauho, and N. A. Mortensen, "Slow-light enhanced absorption in a hollow-core fiber," Opt. Express 18, 14270-14279 (2010).

6. M. Kolesik, E. M. Wright, and J. V. Moloney, "Simulation of femtosecond pulse propagation in sub-micron diameter tapered fibers," Appl. Phys. B 79, 293-300 (2004).

7. S. Afshar V. and T. M. Monro, "A full vectorial model for pulse propagation in emerging waveguides with subwavelength structures part I: Kerr nonlinearity," Opt. Express 17, 2298-2318 (2009).

8. M. D. Turner, T. M. Monro, and S. Afshar V., "A full vectorial model for pulse propagation in emerging waveguides with subwavelength structures part II: stimulated Raman scattering," Opt. Express 17, 11565-11581 (2009).

9. A. Ferrando, M. Zacares, P. de Cordoba, D. Binosi, and A. Montero, "Forward-backward equations for nonlinear propagation in axially invariant optical systems," Phys. Rev. E 71, 016601 (2005).

10. Y. Mizuta, M. Nagasawa, M. Ohtani, and M. Yamashita, "Nonlinear propagation analysis of few-optical-cycle pulses for subfemtosecond compression and carrier envelope phase effect," Phys. Rev. A 72, 063802 (2005).

11. J. Lægsgaard, "Mode profile dispersion in the generalised nonlinear Schrödinger equation," Opt. Express 15, 16110-16123 (2007).

12. M. Kolesik and J. V. Moloney, "Nonlinear optical pulse propagation simulation: from Maxwell's to unidirectional equations," Phys. Rev. E 70, 036604 (2004).

13. M. Soljačić, E. Lidorikis, M. Ibanescu, S. Johnson, J. Joannopoulos, and Y. Fink, "Optical bistability and cutoff solitons in photonic bandgap fibers," Opt. Express 12, 1518-1527 (2004).

14. G. P. Agrawal, Nonlinear Fiber Optics (Academic, 2007).

15. K. J. Blow and D. Wood, "Theoretical description of transient stimulated Raman scattering in optical fibers," IEEE J. Quantum Electron. 25, 2665-2673 (1989).

16. J. Lægsgaard, N. A. Mortensen, and A. Bjarklev, "Mode areas and field energy distribution in honeycomb photonic bandgap fibers," J. Opt. Soc. Am. B 20, 2037-2045 (2003).

17. S. G. Johnson and J. D. Joannopoulos, "Block-iterative frequency-domain methods for Maxwell's equations in a planewave basis," Opt. Express 8, 173-190 (2001).

18. J. Lægsgaard, A. Bjarklev, and S. E. B. Libori, "Chromatic dispersion in photonic crystal fibers: fast and accurate scheme for calculation,” J. Opt. Soc. Am. B 20, 443-448 (2003).

19. N. A. Mortensen and M. D. Nielsen, "Modeling of realistic cladding structures for air-core photonic bandgap fibers," Opt. Lett. 29, 349-351 (2004).

20. W. H. Press, B. P. Flannery, S. A. Teukolsky, and W. T. Vetterling, Numerical Recipes in Fortran $7 \%$ (Cambridge University, 2001).

21. A. Tuniz, G. Brawley, D. J. Moss, and B. J. Eggleton, "Twophoton absorption effects on Raman gain in single mode $\mathrm{As}_{2} \mathrm{Se}_{3}$ chalcogenide glass fiber," Opt. Express 16, 18524-18534 (2008). 